\title{
Brain Imaging Evidence of Preclinical Alzheimer's Disease in Normal Aging
}

\author{
William Jagust, MD, ${ }^{1-3}$ Amy Gitcho, BA, ${ }^{2}$ Felice Sun, PhD, ${ }^{2}$ Beth Kuczynski, PhD,${ }^{2}$ Dan Mungas, PhD, ${ }^{4}$ \\ and Mary Haan, DrPH
}

\begin{abstract}
Objective: This study was designed to test the hypothesis that baseline glucose metabolism and medial temporal lobe brain volumes are predictive of cognitive decline in normal older people. Methods: We performed positron emission tomography using $\left[{ }^{18} \mathrm{~F}\right]$ fluorodeoxyglucose and structural magnetic resonance imaging at baseline in 60 cognitively normal community-dwelling older subjects who were part of a longitudinal cohort study. Subjects were followed for a mean of 3.8 years, with approximately annual evaluation of global cognition (the Modified Mini-Mental State Examination) and episodic memory (delayed recall). Baseline brain volumes and glucose metabolism were evaluated in relation to the rate of change in cognitive test scores. Results: Six subjects developed incident dementia or cognitive impairment (converters). Baseline positron emission tomography scans showed regions in left and right angular gyrus, left mid-temporal gyrus, and left middle frontal gyrus that predicted the rate of change on the Modified Mini-Mental State Examination $(p<0.001)$. The left hemisphere temporal and parietal regions remained significant when converters were excluded. Both hippocampal $(p=0.03)$ and entorhinal cortical volumes $(p=0.01)$ predicted decline on delayed recall over time, and entorhinal cortical volumes remained significant when converters were excluded $(p=0.02)$. These brain volumes did not predict Modified Mini-Mental State Examination decline. Interpretation: These results indicate that temporal and parietal glucose metabolism predict decline in global cognitive function, and medial temporal brain volumes predict memory decline in normal older people. The anatomical location of these findings suggests detection of preclinical Alzheimer's disease pathology.
\end{abstract}

Ann Neurol 2006;59:673-681

Improved understanding of the cellular and molecular basis of Alzheimer's disease (AD) has generated optimism concerning the eventual development of interventions that may ultimately halt or reverse disease progression. This, in turn, has led to an effort to diagnose $\mathrm{AD}$ at the earliest possible stage. Developments in functional, molecular, and structural brain imaging have provided methods that may be useful in the preclinical and presymptomatic detection of $\mathrm{AD}$. Positron emission tomography (PET), structural magnetic resonance imaging (MRI), and functional MRI have been used to detect alterations in the brains of individuals who may be at risk for $\mathrm{AD}$ but who have not yet developed it.

Studies using imaging to detect early evidence of AD are generally based on the preferential atrophy or functional change of specific brain regions in the disease. For example, it is well recognized that medial temporal lobe structures involved in learning and memory, such as the hippocampus and entorhinal cortex, are affected early by $\mathrm{AD}$ pathology. ${ }^{1,2}$ Consequently, atrophy of the hippocampus and entorhinal cortex are the earliest changes in brain structure that have been detected with MRI. ${ }^{3,4}$ Similarly, PET scanning using the glucose metabolic tracer $\left[{ }^{18} \mathrm{~F}\right]$ fluorodeoxyglucose (FDG) has shown reductions in glucose utilization in temporal, parietal, and posterior cingulate cortices that occur relatively early in the course of $\mathrm{AD}$ and that may have clinical diagnostic utility. ${ }^{5,6}$

Several models for the investigation of preclinical $\mathrm{AD}$ have been used. One approach evaluates individuals defined as having amnestic mild cognitive impairment (MCI), a condition marked by memory impairment with preserved daily function and without evidence of dementia that is associated with a substantial risk for development of $\mathrm{AD}$ over time. ${ }^{7}$ In patients with MCI, smaller hippocampal and entorhinal cortical volumes are associated with more rapid progression to
From the ${ }^{1}$ School of Public Health; ${ }^{2}$ Helen Wills Neuroscience Institute; ${ }^{3}$ Lawrence Berkeley National Laboratory, University of California, Berkeley; ${ }^{4}$ Department of Neurology, School of Medicine, University of California, Davis, CA; and ${ }^{5}$ Department of Epidemiology, School of Public Health, University of Michigan, Ann Arbor, MI.

Received Jul 15, 2005, and in revised form Dec 1. Accepted for publication Dec 13, 2005.
Published online Feb 8, 2006 in Wiley InterScience (www.interscience.wiley.com). DOI: 10.1002/ana.20799

Address correspondence to Dr Jagust, Helen Wills Neuroscience Institute, 132 Barker Hall, University of California, Berkeley, CA 94720. E-mail: jagust@berkeley.edu 
AD. ${ }^{8-11}$ Similarly, individuals with MCI who subsequently convert to dementia have lower temporoparietal glucose metabolism seen with FDG-PET. ${ }^{12,13}$ Another method of identifying those at risk for $A D$ involves the study of individuals who have genetic risk factors for the disease. The apolipoprotein $\mathrm{E}(A p o E)$ gene is a well-recognized risk factor for the development of $\mathrm{AD}$, with a single copy of the $\varepsilon 4$ allele conferring an increased risk for $\mathrm{AD}$ that is greater still in those who are homozygous. FDG-PET has identified metabolic lesions similar to those seen in $\mathrm{AD}$ patients and $\mathrm{MCI}$ converters in the temporal, parietal, and posterior cingulate cortices of normal middle-aged and young individuals with one or two copies of the $\varepsilon 4$ allele. $^{14-16}$

Although approaches investigating MCI patients or those with the ApoE $\varepsilon 4$ allele have been useful, they have limitations. MCI patients have cognitive deficits that can be difficult to distinguish from $\mathrm{AD}$, and they may also have relatively advanced Alzheimer's neuropathology. ${ }^{17}$ Thus, these individuals, although preclinical in the sense that they do not have $\mathrm{AD}$, are not presymptomatic. Individuals who have the $\varepsilon 4$ allele may be asymptomatic, but it is not clear whether they will develop $\mathrm{AD}$; therefore, they may not be truly preclinical. Furthermore, such $\varepsilon 4$ carriers may not be representative of the full spectrum of $\mathrm{AD}$, because many cases of $\mathrm{AD}$ occur without this genetic background. The prediction of cognitive decline in older people with neither cognitive loss nor genetic risks would provide evidence of $\mathrm{AD}$ that is earlier than is currently available.

From 1998 to 1999 we recruited a cohort of Latino individuals aged 60 and older who were living in the California Central Valley with the aim of identifying prevalence, incidence, and risk factors for dementia and cognitive decline. The Sacramento Area Latino Study on Aging (SALSA) is continuing follow up through 2008, including the ascertainment of longitudinal cognitive function and the development of incident cognitive dysfunction and dementia. Shortly after the study began, a substudy that utilized imaging with FDG-PET and MRI was initiated. We now report the results of baseline FDG-PET and MRI in predicting the subsequent rate of cognitive decline in the subjects recruited to the imaging substudy.

\section{Subjects and Methods Subjects}

The SALSA project recruited 1,789 Latino individuals (primarily Mexican American individuals) aged 60 to 100. Detailed methods for subject sampling, screening, and evaluation have been described previously. ${ }^{18}$ In brief, census tracts with a high proportion of eligible participants (Latino individuals older than 60) were identified, and individuals were contacted by mail, telephone, and door-to-door recruitment.
Although the ascertainment of cognitive impairment and dementia utilized a multistage approach beginning with screening instruments and followed by more comprehensive neuropsychological testing for those who did not pass the screening stage, subjects participating in the imaging substudy underwent the full neuropsychological battery at enrollment. This consisted of a test of global cognitive function, the Modified Mini-Mental State Examination (3MSE) ${ }^{19}$ the Spanish-English Neuropsychological Assessment Scales (SENAS), ${ }^{20}$ and the informant questionnaire on cognitive decline in the elderly (IQCODE). ${ }^{21}$ The SENAS is a multidimensional neuropsychological test battery with normative data in both English and Spanish that is psychometrically matched across individual scales and both languages. This battery contains tests of verbal episodic memory, verbal and nonverbal semantic memory, verbal attention, verbal abstraction, and visual-perceptual ability. The verbal episodic memory subscale was a 15 -word list with 5 learning trials and a delayed recall (DelRec) condition that followed reading a distractor list. All subjects also had a full medical history, had measurement of physical function, answered detailed questionnaires about lifestyle and depressive symptoms, and had a laboratory evaluation that included measures of blood glucose, lipids, blood pressure, and ApoE genotyping of buccal cell DNA using a modified polymerase chain reaction technique. Procedures for obtaining informed consent were approved by the institutional review boards of all participating universities.

After administration of the neuropsychological battery, subjects underwent clinical neurological examination by a physician if they scored below the 10th percentile on one or more SENAS tests and had an IQCODE score below the 20th percentile. After neurological evaluation, dementia or cognitive impairment was adjudicated at a multidisciplinary consensus conference as described previously. ${ }^{18}$ Criteria for dementia required clinically significant impairment in two or more cognitive domains that indicated decline from premorbid function and clinically significant impairment of independent functioning. Individuals with impairment in one cognitive domain or whose cognitive decline was generalized but mild and did not impair functioning were judged to have cognitive impairment, no dementia (CIND). Follow-up assessments are continuing, with all enrolled participants receiving the $3 \mathrm{MSE}$ and the verbal episodic memory test at reevaluations occurring approximately annually. A full neuropsychological evaluation leading to dementia ascertainment is triggered if an individual demonstrates a decline of 8 or more points on the 3MSE with a score of 77 or less, or drops 3 or more points on the DelRec with a score of 5 or less. We have reported previously that our procedures for detecting prevalent dementia had a sensitivity of $100 \% .{ }^{18}$

Subjects with diagnoses of normal cognition, CIND, or dementia were recruited to the imaging substudy from the larger cohort. For this report, we restricted our evaluations to subjects who were cognitively normal at baseline, had at least two follow-up home visits, and underwent both PET and MRI. Of the 93 subjects who underwent both FDG-PET scanning and MRI, we excluded 20 who were cognitively impaired or demented at baseline. An additional 12 subjects were excluded because they had fewer than 3 evaluations, and 1 subject was excluded because of technical factors re- 
lated to the scan, leaving a total of 60 subjects who were included in these analyses.

\section{Imaging Procedures}

The mean time between entry into the study and PET scanning was 11.4 months (standard deviation [SD], 6.3), and the mean time between MRI and PET was 12 days (SD, 17). MRI utilized a GE Signa system (General Electric, Milwaukee, WI) with a sagittal fast spin-echo localizer, an axial oblique double spin-echo sequence, and a T1-weighted coronal three-dimensional spoiled gradient recalled echo inversion recovery prepped pulse sequence (TE: $1.9 \mathrm{msec}$; flip angle: 20 degrees; field of view: $24 \times 24 \times 18.6 \mathrm{~cm}$; 124 contiguous slices; slice thickness, $1.6 \mathrm{~mm}$; matrix $256 \times$ 256). This spoiled gradient recalled echo sequence was used for determination of hippocampal and entorhinal cortical volume. PET imaging was performed using a Siemens-CTI ECAT EXACT (model 921) 47-section scanner (Siemens Medical Systems, South Iselin, NJ) in two-dimensional mode approximately 30 minutes after the injection of 5 to $10 \mathrm{mCi}$ of FDG. PET data were acquired for 40 minutes and corrected with a 20-minute transmission scan obtained with a rotating ${ }^{68} \mathrm{Ge}$ source. Images were reconstructed using standard two-dimensional filtered backprojection.

Hippocampal and entorhinal cortical volume measurement has been previously described in detail and was done by manually outlining the regions by a single operator with high intrarater reliability. ${ }^{22}$ The hippocampus included the hippocampal subfields, dentate gyrus, and subicular complex in its full rostrocaudal extent from the alveus to the fornix. The entorhinal cortex was defined as the gray matter of the parahippocampal gyrus rostral to the MR slice containing the lateral geniculate nucleus and caudal to the limen insulae. All brain volumes were normalized to the total intracranial volume to control for differences in head size, and left and right hemispheric volumes were averaged unless otherwise noted.

\section{Statistical Analysis}

COGNITIVE CHANGE. A measure of cognitive change was defined for each participant for the 3MSE and DelRec tests by fitting a straight line to the serial test scores. Because the PET or MRI scans were performed at varying intervals after the initial cognitive testing, we estimated the 3MSE and DelRec scores at the date of imaging by fitting a straight line between the initial and second cognitive test scores and estimating the score at the time of the PET. These scores are used as the baseline cognitive measure at the time of PET or MRI. We then used this score, combined with all subsequent test scores available for the subject, to fit a straight line with a slope that described the rate of change on each test. Slopes were thus positive if the scores improved, or negative if they declined. Incidence rates for the development of cognitive impairment or dementia were calculated using the cumulative incidence density function as a ratio of the total number of cases and the cumulative person-time of follow-up.

POSITRON EMISSION TOMOGRAPHY AND MAGNETIC RESONANCE IMAGING ANALYSIS. PET images were first analyzed with a voxel-based approach using Statistical Parametric Mapping 2 (SPM2; Wellcome Department of Cognitive
Neurology, Functional Imaging Laboratory, London, United Kingdom). Images were normalized to a common stereotaxic coordinate system defined by the Montreal Neurological Institute atlas, smoothed with a Gaussian filter with $16 \mathrm{~mm}$ full-width at half-maximum, and normalized to mean global activity. The general linear model was then employed using an approach in which all individual subject's cognitive test slopes were regressed on each voxel across all subjects. Significant associations were defined as clusters of at least 100 voxels significant at $p$ less than 0.001 . We confined our analyses to positive associations; that is, brain regions in which lower glucose metabolism was associated with faster decline. The peak values of these clusters are reported as $x, y, z$ coordinates in Montreal Neurological Institute space.

A second analysis used a multivariate regression approach to evaluate relations between the MR volumes and rates of cognitive change and to investigate whether other potential variables might influence the relations between imaging variables and cognitive change. We performed a series of analyses regressing potential variables against the 3MSE and DelRec slopes. These variables were baseline test scores (3MSE for the 3MSE slope and DelRec for the DelRec slope), baseline functional score (IQCODE), age, education, sex, duration of follow-up, and presence of an ApoE $\varepsilon 4$ allele. Final regression models were then constructed to incorporate any variable that was significantly associated with each cognitive test slope, together with either the medial temporal lobe brain volume (hippocampus or entorhinal cortex) or the PET counts. These PET counts were defined as clusters of voxels that were significantly associated with change scores on the Statistical Parametric Mapping analysis. This was accomplished by extracting globally normalized PET counts from these brain regions using a masking procedure implemented with MarsBaR (MRC Cognition and Brain Sciences Unit, Cambridge, United Kingdom).

In a third analysis, we investigated relations between cognitive change and glucose metabolism using predefined regions of interest on PET medial temporal lobe volumes because of results showing that spatially transforming atrophied brains results in poor overlap of medial temporal brain regions that might result in loss of statistical power. ${ }^{23}$ The regions of interest that were defined on the MR images were superimposed on coregistered PET data for each subject using methods reported previously. ${ }^{24,25}$ Atrophy correction using a two-component technique (brain/cerebrospinal fluid) was applied to the PET data, ${ }^{26}$ and counts were extracted and normalized to whole-brain metabolism. These count rates were then evaluated in regression models as defined earlier.

\section{Results}

The 60 subjects were followed for an average of 3.8 years (median, 4.0; range, 1.5-5.1; SD, 0.9), during which time 5 subjects developed CIND and 1 subject developed dementia that was diagnosed as AD (converters). Four subjects withdrew or were lost to followup, and four died; but all of these individuals contributed at least 1.5 years of observation (mean, 3.1 years) and two follow-up visits. The crude incidence rate for CIND and dementia was 26 in 1,000 person-years. 
Characteristics of the subjects are provided in Table 1 , together with a comparison with the entire nondemented SALSA cohort characteristics at baseline. The subjects in the imaging group were significantly more educated than the entire nondemented cohort, although both groups had low education levels. In general, the imaged subjects scored slightly higher on all of the neuropsychological tests than the rest of the cohort, with the exception of DelRec, for which they scored slightly lower. There were no differences in IQCODE, indicating similar levels of daily function, and no differences in the distribution of sex or ApoE $\varepsilon 4$ gene frequency, which was uncommon in both groups. In addition, $29 \%$ of the subjects had type 2 diabetes, as indicated by a medical diagnosis or prescription for hypoglycemic agents, and $11 \%$ had a medical history of stroke, comparable with rates of $32.7 \%$ and $9.5 \%$, respectively, in the entire SALSA cohort.

Each subject's longitudinal performance on the 3MSE and DelRec tests are shown in Figure 1. Most subjects had stable $3 \mathrm{MSE}$ scores over time, although scores for many subjects did decline. The mean and median 3MSE slopes were -0.37 and 0.08 point/year, respectively. On the DelRec score, subjects showed both improvement and decline in performance over time, with mean and median for the slope of -0.01 and -0.13 point/year. A single subject showed the fastest decline on both tests, with a decline of -11.6 points/year on the 3MSE from a starting score of 92 , and a decline of 2.29 points/year on the DelRec from a starting score of 8 . This subject developed CIND in 2.6 years.

Results for the Statistical Parametric Mapping analysis correlating the 3MSE slopes with glucose metabolism are shown in Figure 2. Brain regions that were highly positively correlated $(p<0.001)$ with the
3MSE slopes were in the right and left angular gyri, left mid-temporal gyrus, and left middle frontal gyrus (Table 2). In these regions, lower glucose metabolism was associated with faster cognitive decline. No brain regions on the medial surfaces were associated with 3MSE change. To evaluate whether subjects with early dementia might be contributing to these PET results, we performed the analysis excluding the six subjects who converted to CIND or dementia. Results for this analysis (see Table 2) show significant associations maintained in left angular and middle temporal gyri. There were no significant associations between glucose metabolism and baseline score on the 3MSE.

Correlational analysis between the baseline PET and the DelRec slope showed a small region within the left superior occipital cortex $(-26,-72,32)$ where decreased glucose metabolism was associated with faster DelRec decline. This region was no longer significant when the converters were excluded.

In a series of bivariate analyses, none of the potential confounding variables test scores (baseline 3MSE, baseline IQCODE, age, education, sex, duration of followup, and presence of an ApoE $\varepsilon 4$ allele) was related to the 3MSE slope. Therefore, the PET count rates were extracted from each significant region and run in bivariate regressions with the 3MSE slope alone. The coefficients were all highly significant $(p<0.0006)$ with $R^{2}$ values for the associations of 0.18 for the right parietal region, 0.22 for the left temporal region, and 0.25 for the left parietal region.

Results of linear regression analyses for the association between the DelRec slope and medial temporal lobe brain volumes are shown in Table 3. Two models were assessed, one for entorhinal cortex and one for hippocampus. There was no association between either hippocampal or entorhinal cortical volume and the

Table 1. Subject Characteristics

\begin{tabular}{lccc}
\hline Characteristics & $\begin{array}{c}\text { This Cohort }(\mathrm{N} \\
=60)\end{array}$ & $\begin{array}{c}\text { All Nondemented } \\
\text { Subjects in SALSA }\end{array}$ \\
\hline Mean age (SD), years & $69.5(5.8)$ & $70.2(6.8)$ & \\
Mean education (SD), years & $9.8(5.1)$ & $7.2(5.3)$ & 0.33 \\
Mean baseline 3MSE score (SD) & $89.2(7.5)$ & $85.8(11.5)$ & 0.0003 \\
Mean baseline DelRec score (SD) & $7.9(3.0)$ & $8.7(2.9)$ & 0.02 \\
Mean verbal semantic memory score (SD) & $10.4(3.4)$ & $8.7(4.2)$ & 0.04 \\
Mean nonverbal semantic memory score (SD) & $10.1(4.0)$ & $8.5(4.0)$ & 0.001 \\
Mean verbal attention score (SD) & $9.6(4.5)$ & $8.8(3.8)$ & 0.02 \\
Mean verbal abstraction score (SD) & $10.3(3.3)$ & $3.9(4.5)$ & 0.06 \\
Mean visual-perceptual score (SD) & $11.3(3.9)$ & $3.17(0.25)$ & 0.03 \\
IQCODE & $3.11(0.24)$ & 59 & 0.10 \\
Female subjects, \% & 57 & 3.3 & 0.81 \\
ApoE $\varepsilon 4$ gene frequency, \% & 5 & 0.11 \\
\hline
\end{tabular}

${ }^{a}$ For age, education, baseline Modified Mini-Mental State Examination (3MSE), apolipoprotein E (ApoE), sex, and delayed recall (DelRec), N was between 1,584 and 1,602. For remaining neuropsychological scores and informant questionnaire on cognitive decline in the elderly (IQCODE), $\mathrm{N}$ was 480 to 491.

SALSA $=$ Sacramento Area Latino Study on Aging; SD = standard deviation. 


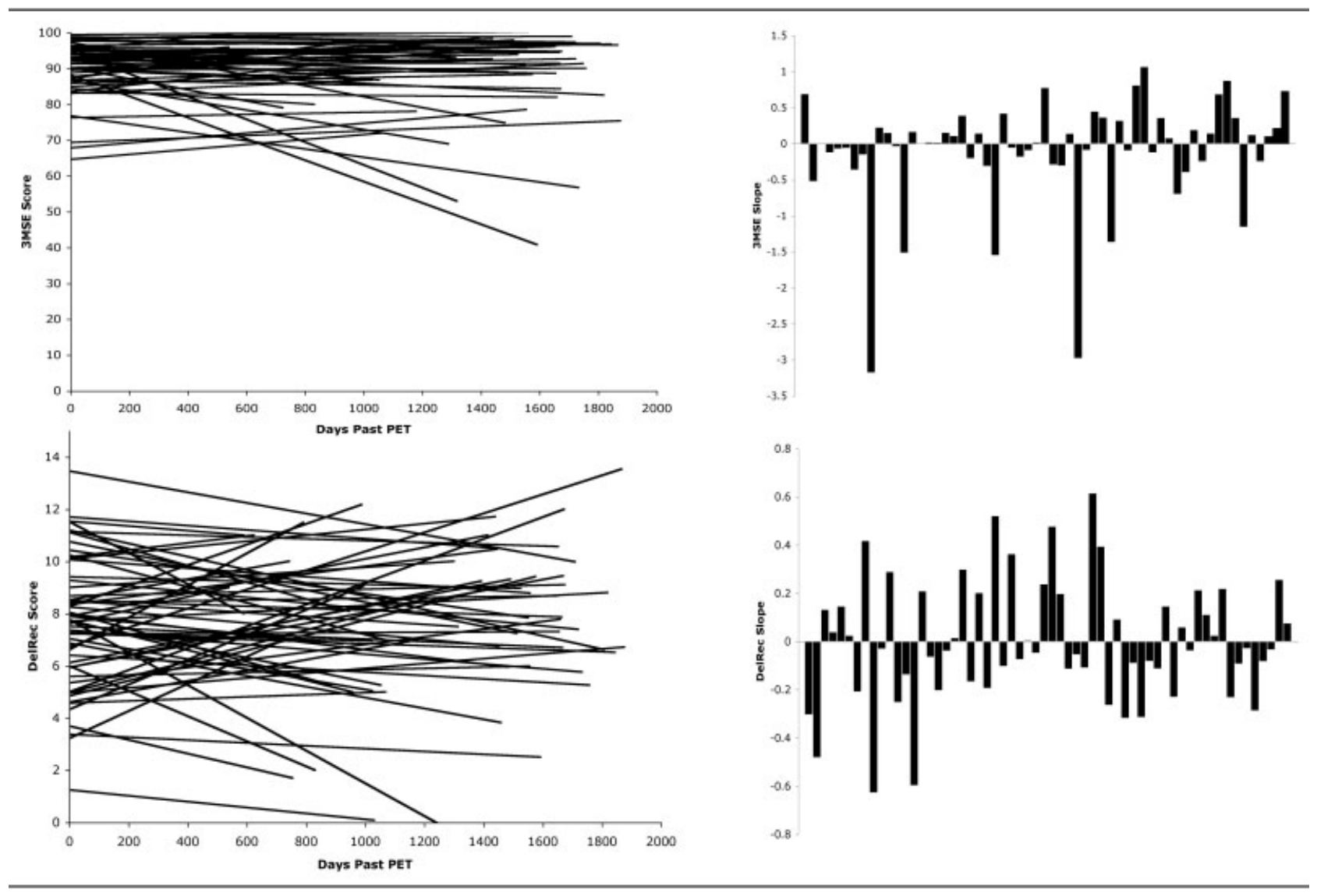

Fig 1. Rates of change of cognitive test scores. (Top left) Change in the Modified Mini-Mental State Examination (3MSE) over time. Time $=0$ is set to the date of the positron emission tomography (PET) scan. (Top right) Individual rates of change on the $3 M S E$ (slope of scores vs time); positive values indicate improvement and negative values indicate decline. (Bottom left) Change in the delayed recall score (DelRec) over time, with time $=0$ the date of the PET scan. (Bottom right) Individual rates of change on the DelRec (slope of scores vs time); positive values indicate improvement and negative values indicate decline.

3MSE slope. Both hippocampal and entorhinal cortical volumes were associated with the DelRec slope. The only other variable that was associated with the DelRec slope was the DelRec baseline score, which was negatively associated with the rate of change, so this was entered into regression models with the brain volumes. These models show that both hippocampal and entorhinal cortical volumes were related to rate of decline on the memory measure when all subjects were included. For hippocampus, each decrease of $1 \mathrm{SD}$ in size was associated with a 0.25 point/year faster decline on the DelRec score, whereas for the entorhinal cortex, each decrease of $1 \mathrm{SD}$ in size was associated with a faster decline of 0.29 point/year. To assess whether early dementia might account for the results and whether the different regions had different sensitivity, we excluded converters in a second set of analyses. When converters were excluded, the hippocampus was no longer a significant predictor of memory decline, but the entorhinal cortex remained significant and the size of the estimate changed little.

When the region of interest approach to PET data analysis was applied to the medial temporal brain regions, there were no significant associations between glucose metabolism in left or right entorhinal cortex and decline on the 3MSE $\left(R^{2}\right.$ values ranged from $0.01-0.04)$. There was a near significant $\left(R^{2}=0.06\right.$; $p=0.07)$ positive association between left hippocampal glucose metabolism and decline on the DelRec.

\section{Discussion}

We found that normal older individuals followed for an average of approximately 4 years demonstrate associations between PET measures of glucose metabolism in the temporal and parietal cortices and the rate of decline on a test of global cognitive function (the 3MSE). Less robust relations were seen between glucose metabolism in the left occipital cortex and memory decline. In addition, volumes of the hippocampus and entorhinal cortex are predictive of decline in memory, but not the 3MSE. Although this effect is partly due to the individuals who converted from normal cognitive function to cognitive impairment or dementia, temporoparietal metabolism predicts the rate of 


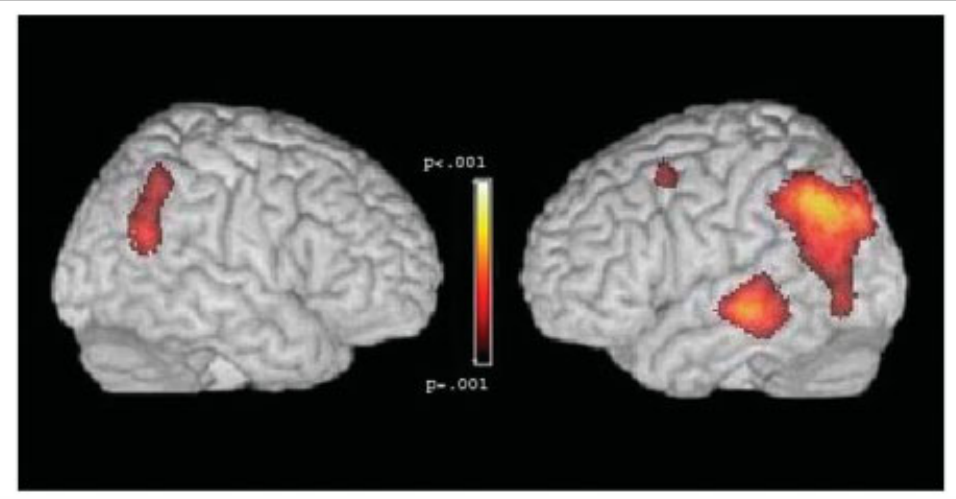

Fig 2. Results of regressions between glucose metabolism and Modified Mini-Mental State Examination (3MSE) decline. Right and left hemispheres of a standard brain with voxels (averaged across all subjects in standard space) showing significant ( $\mathrm{p}<0.001$ ) correlations between glucose metabolism and the 3MSE slope for all 60 subjects in the cohort.

change in the 3MSE, and entorhinal cortex volume predicts the rate of change in memory in subjects who remained cognitively intact. The pattern of glucose metabolism, together with the location of the medial temporal brain regions that are predictive, suggests that these findings are due to the detection of presymptomatic $\mathrm{AD}$.

Previous reports investigating subjects with MCI or the ApoE $\varepsilon 4$ gene have found regional brain abnormalities that are similar in topography to those reported here. In addition, there is some evidence that the severity of abnormality may predict future trajectories in those at-risk groups. Glucose metabolism in parietal and posterior cingulate cortex is predictive of the rate of memory decline in $\varepsilon 4$-positive individuals with mild memory loss (but not in those without this allele) over 2 years. ${ }^{15}$ Functional MRI has shown areas of increased brain activity, presumed due to compensatory mechanisms, in subjects with either the $\varepsilon 4$ allele $^{27}$ or MCI. ${ }^{28}$ In these studies, the degree of increased activation was related to the rate of subsequent memory decline or conversion to $\mathrm{AD}$.
All AD prediction studies grapple with the problem of both defining who will get the disease and selecting individuals who are not already so advanced that this prediction is trivial. The selection of MCI patients or those at genetic risk manage this problem in different ways, neither of which is fully satisfactory. Although subjects in our study had DelRec scores that were lower than that of the entire SALSA cohort, a number of lines of evidence point to the fact that our subjects were not misclassified as normal at baseline. First, the subjects in our study were not judged to be cognitively impaired at baseline, nor did they complain of memory loss or show evidence of functional impairment, and they clearly would meet no criteria for MCI or cognitive loss. Although some of the baseline cognitive scores were low, these scores were not adjusted for education or other variables that have a profound effect in a cohort with a low education level (eg, an unadjusted 3MSE score of 65 for an individual with 2 years of education corresponds to a score of 78 for someone with 12 years of education in this cohort). Education was, however, taken into consideration in dementia ad-

Table 2. Location of Brain Regions Significantly Correlated with Cognitive Decline on the Modified Mini-Mental State Examination

${ }^{a}$ Coordinates of the peak voxel in Montreal Neurological Institute (MNI) Atlas space. 


\begin{tabular}{|c|c|c|c|c|c|c|}
\hline & \multicolumn{3}{|c|}{ All Subjects } & \multicolumn{2}{|c|}{ Converters Excluded } & \multirow[b]{2}{*}{$p$} \\
\hline & Estimate $^{a}$ & Partial $R^{2}$ & $p$ & Estimate $^{\mathrm{a}}$ & Partial $R^{2}$ & \\
\hline \multicolumn{7}{|l|}{ Model 1} \\
\hline Average hippocampal volume & 0.25 & 0.07 & 0.03 & 0.17 & 0.04 & 0.14 \\
\hline DelRec baseline & -0.10 & 0.09 & 0.008 & -0.12 & 0.15 & 0.002 \\
\hline \multicolumn{7}{|l|}{ Model 2} \\
\hline Average entorhinal cortex volume & 0.29 & 0.11 & 0.01 & 0.25 & 0.09 & 0.02 \\
\hline DelRec baseline & -0.09 & 0.09 & 0.01 & -0.12 & 0.15 & 0.002 \\
\hline
\end{tabular}

${ }^{a}$ For brain volumes, estimates reflect the change in delayed recall (DelRec) score/year for each standard deviation change in brain volume. For the DelRec baseline, estimates reflect the change in DelRec score/year for each point on the DelRec baseline.

judication and would not, in any case, affect the calculated rate of change on cognitive tests. Second, baseline memory performance did not explain the associations between imaging measures and cognitive decline. Indeed, the associations between baseline DelRec score and decline was inverse, which could be partly explained by a learning effect. Furthermore, we have previously reported that individuals with low memory scores on the DelRec measure show no decrements in glucose metabolism or hippocampal atrophy. ${ }^{29,30}$ Finally, the subjects most likely to have been misclassified at baseline are those who converted to CIND or dementia. When these subjects were excluded, glucose metabolism in left temporal and parietal cortices and entorhinal cortical brain volumes remained significant predictors of 3MSE and DelRec decline, respectively.

Our data show that PET and MRI have predictive value for cognitive decline but not specifically for $\mathrm{AD}$. However, the location of the temporal and parietal areas that predict decline are the same regions that have been associated with $\mathrm{AD}$ in clinical and pathological studies, ${ }^{6}$ and hippocampal and entorhinal volume reductions are also strongly associated with AD. ${ }^{31}$ The substantial similarity in the findings whether converters were included or excluded from the analyses also suggests that conversion and decline have similar underlying mechanisms.

Some aspects of the associations with glucose metabolism are unusual. The posterior cingulate cortex, a brain region frequently involved in early cases of $\mathrm{AD},{ }^{5}$ was not found to be associated with change in either cognitive score, and the association between DelRec and occipital glucose metabolism was unanticipated. These results could reflect a number of factors including the presence of cerebrovascular disease in this cohort, which may alter the metabolic expression of AD. ${ }^{32}$ The finding of an association with left frontal glucose metabolism, although not characteristically identified as a marker of $\mathrm{AD}$, has also been reported in asymptomatic ApoE4 homozygotes. ${ }^{14}$

The pattern of associations between the PET and
MR measures and the cognitive decline generally reflects what is known about age-related cognitive decline. The global cognitive summary measure was related largely to neocortical changes in glucose metabolism, whereas a measure of episodic memory was related to medial temporal lobe volumes. This dissociation is not likely to be explained by methodological factors and is congruent with the localization of memory function to medial temporal brain regions and localization of a host of other cognitive functions to association neocortex. It is also consistent with other imaging studies of the progression of $\mathrm{AD}$, which indicate that the earliest changes, associated with memory loss, occur in the medial temporal lobes, whereas later stages of the disease, when global cognitive failure supervenes, are associated with neocortical abnormalities. ${ }^{33,34}$ Within the temporal lobe itself there is disagreement about whether the entorhinal cortex or hippocampus is the most sensitive location for the detection of early disease with MRI. ${ }^{35,36}$ However, the entorhinal cortex pathologically is the site that is affected earliest by neurofibrillary change and neuronal loss, ${ }^{2}$ and memory function is related to entorhinal cortical neurofibrillary pathology in normal and MCI subjects. ${ }^{37}$ The finding that hippocampal volumes predicted decline in all subjects but only entorhinal cortex predicted decline when converters were excluded is consistent with the idea that earliest changes are seen in the entorhinal cortex.

In normal older people, some studies have shown cross-sectional associations between the volumes of medial temporal lobe structures and memory function. ${ }^{38,39}$ However, these associations are not always found or may be explained by age. ${ }^{40,41}$ Far less information is available concerning the prediction of decline in normal individuals. The degree of hypometabolism measured in the entorhinal cortex with MR-guided FDG-PET predicted conversion to MCI in a group of normal older subjects, many of whom had memory complaints at baseline. ${ }^{42}$ MR measurement of atrophy rate in the entorhinal cortex, hippocampus, and medial temporal lobe may be capable of predicting memory 
performance, cognitive decline, and conversion to dementia or MCI, but these measurements are made over a number of years. ${ }^{43-45}$

The potential ability to generalize these results deserves comment. Although the study is composed entirely of Latino subjects, there is no clear reason that the results should be any less generalizable than the many existing studies of predominantly Anglo subjects. The subjects in the imaging substudy were reasonably representative of the entire recruited SALSA cohort, and the SALSA cohort was, in turn, representative of the target population of California Central Valley Latinos. ${ }^{18}$ It is possible that the lower education levels and high prevalence of diabetes were related to faster decline in this cohort than might otherwise be seen in more highly educated and healthier cohorts because these factors are associated with cognitive decline, dementia, and neuroanatomical changes. ${ }^{46,47}$ Nevertheless, the incidence rate of CIND and dementia were relatively low and in concert with those reported in previous studies. ${ }^{48-50}$ Overall, the sample did not decline much on the cognitive tests, although both tests have ceiling and floor effects. In addition, the rates of decline on the 3MSE were comparable with those reported in other population samples of a similar age. ${ }^{51}$ It appears unlikely that our results are due to unusually rapid progression of decline, misclassification of mildly demented subjects as normal, or inclusion of unusually medically ill individuals. Rather, this study provides evidence that both metabolic and structural brain alterations predictive of cognitive decline are detectable in normal older people.

This study was supported by the NIH (National Institute on Aging, AG12975, M.H., AG10220, D. M., National Institute of Diabetes and Digestive and Kidney Diseases, DK60753, M.H.).

\section{References}

1. Hyman BT, Van Hoesen GW, Damasio AR, Barnes CL. Alzheimer's disease: cell-specific pathology isolates the hippocampal formation. Science 1984;225:1168-1170.

2. Braak H, Braak E. Neuropathological staging of Alzheimerrelated changes. Acta Neuropathol 1991;82:239-259.

3. Jack CR, Petersen RC, Xu UC, et al. Medial temporal atrophy on MRI in normal aging and very mild Alzheimer's disease. Neurology 1997;49:786-794.

4. Dickerson BC, Goncharova I, Sullivan MP, et al. MRI-derived entorhinal and hippocampal atrophy in incipient and very mild Alzheimer's disease. Neurobiol Aging 2001;22:747-754.

5. Minoshima S, Giordani B, Berent S, et al. Metabolic reduction in the posterior cingulate cortex in very early Alzheimer's disease. Ann Neurol 1997;42:85-94.

6. Silverman DH, Small GW, Chang CY, et al. Positron emission tomography in evaluation of dementia: regional brain metabolism and long-term outcome. JAMA 2001;286:2120-2127.

7. Petersen RC, Smith GE, Waring SC, et al. Mild cognitive impairment: clinical characterization and outcome. Arch Neurol 1999;56:303-308.
8. Jack CR, Petersen RC, Xu YC, et al. Prediction of AD with MRI-based hippocampal volume in mild cognitive impairment. Neurology 1999;52:1397-1403.

9. Killiany RJ, Gomez-Isla T, Moss M, et al. Use of structural magnetic resonance imaging to predict who will get Alzheimer's disease. Ann Neurol 2000;47:430-439.

10. Korf ESC, Wahlund LO, Visser PJ, Scheltens P. Medial temporal lobe atrophy on MRI predicts dementia in patients with mild cognitive impairment. Neurology 2004;63:94-100.

11. deToledo-Morrell L, Stoub TR, Bulgakova M, et al. MRIderived entorhinal volume is a good predictor of conversion from MCI to AD. Neurobiol Aging 2004;25:1197-1203.

12. Arnaiz E, Jelic V, Almkvist $\mathrm{O}$, et al. Impaired cerebral glucose metabolism and cognitive functioning predict deterioration in mild cognitive impairment. Neuroreport 2001;12:851-855.

13. Chetelat G, Desgranges B, De La Sayette V, et al. Mild cognitive impairment: can FDG-PET predict who is to rapidly convert to Alzheimer's disease? Neurology 2003;60:1374-1377.

14. Reiman EM, Caselli RJ, Yun LS, et al. Preclinical evidence of Alzheimer's disease in persons homozygous for the e4 allele for apolipoprotein E. N Engl J Med 1996;334:752-758.

15. Small GW, Ercoli LM, Silverman DH, et al. Cerebral metabolic and cognitive decline in persons at genetic risk for Alzheimer's disease. Proc Natl Acad Sci U S A 2000;97: 6037-6042.

16. Reiman EM, Chen K, Alexander GE, et al. Functional brain abnormalities in young adults at genetic risk for late-onset Alzheimer's dementia. Proc Natl Acad Sci U S A 2004;101: 284-289.

17. Price JL, Morris JC. Tangles and plaques in nondemented aging and "preclinical" Alzheimer's disease. Ann Neurol 1999;45: 358-368.

18. Haan MN, Mungas DM, Gonzalez HM, et al. Prevalence of dementia in older Latinos: the influence of type 2 diabetes mellitus, stroke and genetic factors. J Am Geriatr Soc 2003;51: 169-177.

19. Teng EL, Chui HC. The Modified Mini-Mental State (3MS) examination. J Clin Psychiatry 1987;48:314-318.

20. Mungas D, Reed BR, Marshall SC, Gonzalez HM. Development of psychometrically matched English and Spanish language neuropsychological tests for older persons. Neuropsychology 2000;14:209-223.

21. Del-Ser T, Morales JM, Barquero MS, et al. Application of a Spanish version of the "Informant Questionnaire on Cognitive Decline in the Elderly" in the clinical assessment of dementia. Alzheimer Dis Assoc Disord 1997;11:3-8.

22. Petkov CI, Wu CC, Eberling JL, et al. Correlates of memory function in community-dwelling elderly: the importance of white matter hyperintensities. J Int Neuropsychol Soc 2004;10: 371-381.

23. Mosconi L, Tsui WH, De Santi S, et al. Reduced hippocampal metabolism in MCI and AD: automated FDG-PET image analysis. Neurology 2005;64:1860-1867.

24. Klein GJ, Teng X, Jagust WJ, et al. A methodology for specifying PET VOIs using multi-modality techniques. IEEE Trans Med Imaging 1997;16:405-415.

25. Kwan LT, Reed BR, Eberling JL, et al. Effects of subcortical cerebral infarction on cortical glucose metabolism and cognitive function. Arch Neurol 1999;56:809-814.

26. Meltzer CC, Leal JP, Mayberg HS, et al. Correction of PET data for partial volume effects in human cerebral cortex by MR imaging. J Comput Assist Tomogr 1990;14:561-570.

27. Bookheimer SY, Strojwas MH, Cohen MS, et al. Patterns of brain activation in people at risk for Alzheimer's disease. N Engl J Med 2000;343:450-456. 
28. Dickerson BC, Salat DH, Bates JF, et al. Medial temporal lobe function and structure in mild cognitive impairment. Ann Neurol 2004;56:27-35.

29. Jagust WJ, Eberling JL, Wu CC, et al. Brain function and cognition in a community sample of elderly Latinos. Neurology 2002;59:378-383.

30. Wu CC, Mungas D, Petkov CI, et al. Brain structure and cognition in a community sample of elderly Latinos. Neurology 2002;59:383-391.

31. Jack CR, Dickson DW, Parisi JE, et al. Antemortem MRI findings correlate with hippocampal neuropathology in typical aging and dementia. Neurology 2002;58:750-757.

32. DeCarli C, Grady CL, Clark CM, et al. Comparison of positron emission tomography, cognition and brain volume in Alzhiemer's disease with and without severe abnormalities of white matter. J Neurol Neurosurg Psychiatry 1996;60: $158-167$.

33. De Santi S, de Leon MJ, Rusinek H, et al. Hippocampal formation glucose metabolism and volume losses in MCI and AD. Neurobiol Aging 2001;22:529-539.

34. Smith AD. Imaging the progression of Alzheimer pathology through the brain. Proc Natl Acad Sci U S A 2002;99: 4135-4137.

35. Xu Y, Jack CR Jr, O’Brien PC, et al. Usefulness of MRI measures of entorhinal cortex versus hippocampus in AD. Neurology 2000;54:1760-1767.

36. Killiany RJ, Hyman BT, Gomez-Isla T, et al. MRI measures of entorhinal cortex vs hippocampus in preclinical AD. Neurology 2002;58:1188-1196.

37. Guillozet AL, Weintraub S, Mash DC, Mesulam MM. Neurofibrillary tangles, amyloid, and memory in aging and mild cognitive impairment. Arch Neurol 2003;60:729-736.

38. Golomb J, de Leon MJ, Kluger A, et al. Hippocampal atrophy in normal aging: an association with recent memory. Arch Neurol 1993;50:967-973.

39. Walhovd KB, Fjell AM, Reinvang I, et al. Size does matter in the long run: hippocampal and cortical volume predict recall across weeks. Neurology 2004;63:1193-1197.
40. Raz N, Gunning-Dixon FM, Head D, et al. Neuroanatomical correlates of cognitive aging: evidence from structural magnetic resonance imaging. Neuropsychology 1998;12:95-114.

41. Van Petten C, Plante E, Davidson PS, et al. Memory and executive function in older adults: relationships with temporal and prefrontal gray matter volumes and white matter hyperintensities. Neuropsychologia 2004;42:1313-1335.

42. de Leon MJ, Convit A, Wolf OT, et al. Prediction of cognitive decline in normal elderly subjects with 2-[(18)F]fluoro-2-deoxyD-glucose/poitron-emission tomography (FDG/PET). Proc Natl Acad Sci U S A 2001;98:10966-10971.

43. Rusinek H, De Santi S, Frid D, et al. Regional brain atrophy rate predicts future cognitive decline: 6-year longitudinal MR imaging study of normal aging. Radiology 2003;229:691-696.

44. Rodrigue KM, Raz N. Shrinkage of the entorhinal cortex over five years predicts memory performance in healthy adults. J Neurosci 2004;24:956-963.

45. Jack CR, Shiung MM, Gunter JL, et al. Comparison of different MRI brain atrophy rate measures with clinical disease progression in AD. Neurology 2004;62:591-600.

46. Convit A, Wolf OT, Tarshish C, de Leon MJ. Reduced glucose tolerance is associated with poor memory performance and hippocampal atrophy among normal elderly. Proc Natl Acad Sci U S A 2003;100:2019-2022.

47. Wu JH, Haan MN, Liang J, et al. Impact of diabetes on cognitive function among older Latinos: a population-based cohort study. J Clin Epidemiol 2003;56:686-693.

48. Solfrizzi V, Panza F, Colacicco AM, et al. Vascular risk factors, incidence of MCI, and rates of progression to dementia. Neurology 2004;63:1882-1891.

49. Ganguli M, Dodge HH, Chen P, et al. Ten-year incidence of dementia in a rural elderly US community population: the MoVIES Project. Neurology 2000;54:1109-1116.

50. Miech RA, Breitner JC, Zandi PP, et al. Incidence of AD may decline in the early 90s for men, later for women: the Cache County study. Neurology 2002;58:209-218.

51. Haan M, Shemanski L, Jagust WJ, et al. The role of APOE e4 in modulating effects of other risk factors for cognitive decline in elderly persons. JAMA 1999;40-46. 